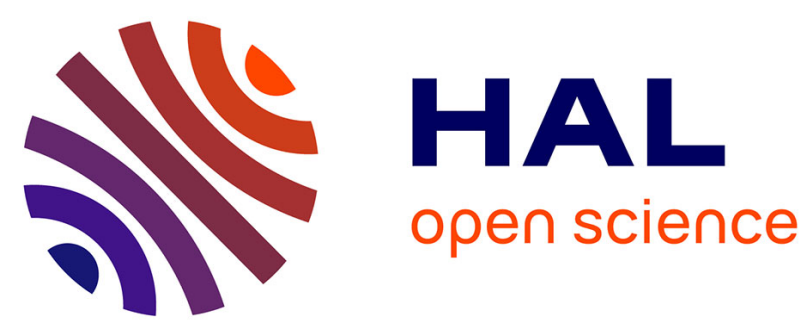

\title{
Quelle marge de manoeuvre faut-il laisser aux utilisateurs d'animations 3D techniques en maintenance aéronautique?
}

David Robin, André Tricot, Pascale Hugues

\section{- To cite this version:}

David Robin, André Tricot, Pascale Hugues. Quelle marge de manoeuvre faut-il laisser aux utilisateurs d'animations 3D techniques en maintenance aéronautique?. 25ème conférence francophone sur l'Interaction Homme-Machine, IHM'13, AFIHM, Nov 2013, Bordeaux, France. $10.1145 / 2534903.2534912$. hal-00877265v2

\section{HAL Id: hal-00877265 \\ https://hal.inria.fr/hal-00877265v2}

Submitted on 6 Nov 2013

HAL is a multi-disciplinary open access archive for the deposit and dissemination of scientific research documents, whether they are published or not. The documents may come from teaching and research institutions in France or abroad, or from public or private research centers.
L'archive ouverte pluridisciplinaire HAL, est destinée au dépôt et à la diffusion de documents scientifiques de niveau recherche, publiés ou non, émanant des établissements d'enseignement et de recherche français ou étrangers, des laboratoires publics ou privés. 


\section{Quelle marge de manœuvre faut-il laisser aux utilisateurs d'animations 3D techniques en maintenance aéronautique ?}

\author{
David Robin \\ CLLE-LTC, Université de Toulouse 2 \\ 5 allées Antonio Machado \\ 31058 Toulouse Cedex 9 \\ david.robin@univ-tlse2.fr
}

\author{
André Tricot \\ CLLE-LTC, Université de Toulouse 2 \\ 5 allées Antonio Machado \\ 31058 Toulouse Cedex 9 \\ andre.tricot@univ-tlse2.fr
}

\author{
Pascale Hugues \\ CTO IW SP IS - Multimodal services \\ EADS Innovation Works 18, rue Marius \\ Terce 31000 Saint Martin du Touch \\ Pascale.Hugues@eads.net
}

\begin{abstract}
RÉSUMÉ
En maintenance aéronautique, l'amélioration de la sécurité passe par une parfaite compréhension d'informations visuelles nécessaires à la réalisation des différentes tâches. Notre étude vise à mesurer de quelles manières des modifications de ces présentations iconographiques affectent leurs utilisations par des techniciens novices. Plus précisément ici, nous avons analysé les effets apportés par une graduation du contrôle du dynamisme sur des documents 3D. Contrairement à notre attente, la version du matériel visuel censée être la plus adaptée à ces tâches de maintenance, produit les moins bonnes performances, sans obtenir en contrepartie d'évaluations favorables de charge cognitive par les utilisateurs.
\end{abstract}

\section{Mots Clés}

Maintenance aéronautique, ergonomie des documents numériques, contrôle du dynamisme, animation 3D.

\section{ACM Classification Keywords}

H.5.2. User interfaces

\section{INTRODUCTION}

La conception des manuels qui délivrent aux techniciens les procédures à suivre pour garantir la navigabilité d'un aéronef, est un enjeu crucial pour les constructeurs aéronautiques. Une erreur de maintenance entraîne non seulement d'importants retards dans un trafic aérien en expansion et des coûts financiers extrêmement élevés, mais aussi des accidents dramatiques. Commanditée par EADS, cette étude participe à la recherche sur les documents procéduraux en explorant la transmission des informations contenues dans leurs illustrations. Leur efficacité dépend du type de contenu à transmettre, du niveau d'interactivité, de l'objectif de l'illustration, de son design et de la variabilité interindividuelle [2]. L'évaluation de cet outil de communication s'est donc

(C) ACM, 2013. This is the author's version of the work. It is posted here by permission of ACM for your personal use. Not for redistribution. The definitive version was published in Actes de la $25^{\text {ième }}$ conférence francophone sur l'Interaction Homme-Machine, 2013.

http://dx.doi.org/10.1145/2534903.2534912 située à l'intersection de l'ergonomie, la psychologie cognitive et la sémiotique. Ce rapprochement a permis d'élaborer les protocoles expérimentaux destinés à évaluer ce matériel visuel spécifique. Nous avons ainsi voulu vérifier si les manipulations des animations $3 \mathrm{D}$ ne sont pas trop exigeantes au détriment de la compréhension et de la restitution des informations transmises pour des opérateurs de maintenance novices.

\section{CONTEXTE DE L'ÉTUDE}

La maintenance aéronautique regroupe l'ensemble des opérations nécessaires à accomplir afin que l'avion remplisse sa fonction de transport dans les conditions de sécurité optimales. Qu'il s'agisse d'une tâche préparatoire ou de réalisation (inspection, dépose, repose), elle comprend des moments de récupération d'information séparés des temps d'exécutions techniques [32]. Pour réaliser ces tâches, le technicien trouve les informations légales dans un document numérique dont l'utilisation est fortement marquée par des contraintes organisationnelles (pression temporelle), environnementales (travail à l'extérieur, bruits, poussières) et de postes (accès au matériel, poids, complexité des équipements etc.). Si les rapports d'incidents montrent que ceux-ci ont des origines multiples, le défaut informationnel est identifié comme cause première pour $71 \%$ des cas [12]. La déficience même du document y est tout aussi importante que la responsabilité de l'opérateur $(46 \%$ des cas d'incidents contre 54\%). Hobbs (2008) montre que ces incidents surviennent lorsque l'opérateur se trompe dans la procédure sans en avoir conscience, qu'il réalise la tâche demandée sans en avoir la connaissance nécessaire, ou bien parce qu'il choisit délibérément de ne pas appliquer la procédure écrite [10]. 80\% des techniciens avouaient déjà s'en être ainsi éloignés au moins une fois l'année précédente en 2002 [11]. L'amélioration de la documentation de maintenance aéronautique vise donc la réduction de ces trois sources accidentogènes.

Conçue par des experts du domaine avec peu de retours des utilisateurs, cette documentation possède les caractéristiques des textes procéduraux comme la fragmentation de l'action établie sous forme de buts à 
atteindre. Les illustrations techniques qui y sont proposées relèvent d'une grande variété (schémas, diagrammes, photos, animations 3D) et assument différentes fonctions: identification de pièces, prise d'information, modélisation de connaissances, mais aussi démonstration esthétique ou création de lien social. Les éléments de ce corpus iconographique peuvent être décrits et comparés par une série de propriétés sémiotiques : iconicité, indicialité, dynamisme, sonorisation, multiplicité, mobilité, taille, etc. [26].

Parmi celles-ci, la capacité de l'opérateur à commander sur la représentation est une propriété transversale. Elle est souvent nommée interactivité, mais ce terme a plusieurs sens $[18,13]$ et renvoie aussi bien à l'interaction (la possibilité de communications entre opérateurs réels et/ou virtuels [19]) qu'à la réactivité du document. Dans notre contexte, cette propriété se limite à la possibilité laissée au lecteur de modifier certains aspects visuels de la présentation, les contenus étant figés pour des raisons légales. Les occurrences les plus fréquentes de cette propriété de contrôle portent sur le dynamisme des représentations à tel point qu'une typologie échelonne la quantité de contrôle à la disposition de l'utilisateur (Tableau 1) [31, 22, 7]. Les premiers niveaux sont souvent amalgamés pour former une situation de «basse interactivité » et opposés empiriquement à l'absence de contrôle [1].

Concernant l'efficacité des multimédias contrôlés, les résultats empiriques sont, comme d'ailleurs pour la question du dynamisme seul, très nuancés. L'apprentissage se révèle meilleur avec l'animation contrôlée (niveau 1 vs. niveau 0 ), si le participant ne gère que le rythme de diffusion, le découpage étant déjà fait [16]. Avec un dispositif comparant des niveaux 0,1 et 2 [30], on n'obtient pas d'effets du contrôle sur la compréhension, mais on observe un temps plus court d'apprentissage [20]. Le bénéfice est dans l'efficience plus que dans l'efficacité [8] et le contrôle de l'animation semble donc être une aide efficace mais provisoire.

Sur des contenus procéduraux, l'expérience fondatrice compare niveaux 2 et 0 à partir de vidéos pour réaliser des nœuds marins de plus en plus complexes [28]. Les participants qui disposaient du contrôle ont appris plus vite, ce qui sous-entend qu'ils ont réussi à gérer la progression des informations, en adaptant leurs ressources cognitives sur les passages de la vidéo en fonction de leurs besoins cognitifs. La reprise de cette expérience $[6,4,23]$, montre cependant que les meilleurs rappels correspondent à la version non interactive.

L'hypothèse généralement admise $[16,17,18,20]$ est donc que le contrôle permet à un apprenant de limiter l'effet de surcharge cognitive dû au flux continu d'informations, en régulant l'information entrante quand celle-ci devient trop coûteuse à traiter. Mais cette gestion de débit informationnel a un coût, prélevé aux dépens de la pérennisation des connaissances acquises via le contact avec le document.

Pour résumer, le contrôle du document visuel peut être bénéfique aux novices du domaine pour la compréhension de connaissances ou d'instructions lorsque :

- le contenu abordé n'est pas trop complexe, $[14,15$, 3].

- l'objectif de cette utilisation est clairement établi [5];

- le contrôle est limité afin de surmonter les limites perceptives induites $[16,21]$.

- le fonctionnement du contrôle se fait oublier en étant intuitif, réactif et bien situé $[20,5]$.

- Le contrôle n'est pas en concurrence avec une activité collaborative [24, 25].

\section{EXPÉRIENCE}

L'objectif de cette expérience est de vérifier auprès de futurs techniciens de maintenance si l'extension des possibilités de commandes sur des animations 3D se traduit par des différences de performances dans la compréhension et la mémorisation des informations véhiculées. Si pour des raisons légales et pratiques (durée de l'épreuve, accès au matériel), de véritables tâches de maintenance in situ ne pouvaient être envisagées, une approche écologique a été privilégiée par l'utilisation de matériel directement issu du manuel de maintenance (Airbus A380) sur la population ciblée des techniciens.

\begin{tabular}{|cl|l|}
\hline Niveaux de contrôle du document & Actions possibles de l'utilisateur \\
\hline $\mathbf{0}$ & $\begin{array}{l}\text { Absence de contrôle } \\
\text { (diffusion linéaire et continue) }\end{array}$ & Suivre (subir) la représentation dynamique ou l'abandonner (éteindre le document). \\
\hline $\mathbf{1}$ & $\begin{array}{l}\text { Contrôle de la lecture } \\
\text { (diffusion linéaire interruptible) }\end{array}$ & $\begin{array}{l}\text { Contrôler la vitesse, la direction (lecture avant / arrière), la continuité (pause, arrêt); } \\
\text { l'utilisateur régule le contenu en fonction de ses compétences cognitives, de ses } \\
\text { connaissances et des contraintes imposées par la tâche [14]. }\end{array}$ \\
\hline $\mathbf{2}$ & $\begin{array}{l}\text { Contrôle de la consultation } \\
\text { (lecture séquentielle, par cellule) }\end{array}$ & $\begin{array}{l}\text { Naviguer jusqu'à un point précis (déplacement dans la séquence, avant et retour, } \\
\text { curseur) }\end{array}$ \\
\hline $\mathbf{3}$ & $\begin{array}{l}\text { Manipulation / Contrôle de la } \\
\text { représentation et des objets représentés }\end{array}$ & $\begin{array}{l}\text { Se déplacer vers les objets ; les transformer (faire disparaitre, changer d'aspects) ; } \\
\text { diriger des rotations, panoramiques et zoom pour atteindre un objet précis du contexte. }\end{array}$ \\
\hline $\mathbf{4}$ & $\begin{array}{l}\text { Simulation / Contrôle de } \\
\text { l'environnement virtuel }\end{array}$ & $\begin{array}{l}\text { Programmer les modèles qui président l'ensemble de la représentation (activités } \\
\text { scientifiques prédictives [21]) }\end{array}$ \\
\hline
\end{tabular}

Tableau 1 : Graduation du contrôle $[7,22]$ 


\section{Participants}

A la demande d'EADS, la population est restreinte aux techniciens de maintenance novices: les participants sont des techniciens qui achèvent leur BTS aéronautique. Leurs capacités visuospatiales sont évaluées (DAT5) pour créer trois groupes de niveaux comparables (Tableau 2). Nous avons aussi évalué, par questionnaire, la fréquence avec laquelle les participants se servent d'images pour communiquer, selon les types d'image et leurs supports technologiques. Cette pratique iconographique peut être considérée comme une forme d'expertise.

A chacun des trois groupes ainsi formés va correspondre un niveau de contrôle des mêmes documents 3D :

- Niveau 1 : l'utilisateur du premier groupe peut arrêter / reprendre le défilement ([pause]). Lorsque l'animation est terminée, il peut la redémarrer.

- Niveau 2 : dans le second groupe, on peut lire / arrêter l'animation à sa guise, se déplacer dans le cours de celleci en utilisant la barre de défilement, ou encore utiliser les touches d'avance et retour rapides.

- Niveau 3: un participant du $3^{\mathrm{e}}$ groupe dispose de toutes les commandes sur le défilement : il peut déplacer la caméra virtuelle (travelling avant / arrière ; panoramique haut / bas et gauche / droite), et zoomer (avant / arrière), sans attendre que l'animation ne le lui propose.

Avant la passation, les modes de contrôle sont explicités. L'ensemble des utilisateurs savent déjà utiliser les boutons de défilement et le curseur; ceux du niveau 3 se font rapidement la main sur les boutons correspondant au déplacement, la rotation et le zoom (au maximum 45s).

\begin{tabular}{|l|c|c|c|}
\hline $\begin{array}{r}\text { Niveau de contrôle } \\
\text { accordé }\end{array}$ & $\begin{array}{c}\text { Limité } \\
(\text { niv .1) }\end{array}$ & $\begin{array}{c}\text { Médium } \\
\text { (niv. 2) }\end{array}$ & $\begin{array}{c}\text { Étendu } \\
\text { (niv.3) }\end{array}$ \\
\hline $\begin{array}{l}\text { Nombre de participants } \\
\text { Âge moyen du groupe } \\
\text { (écart-type) }\end{array}$ & $\begin{array}{c}18 \text { ans } \\
(1,2)\end{array}$ & $\begin{array}{c}19 \text { ans } \\
(1,6)\end{array}$ & $\begin{array}{c}19 \text { ans } \\
(1,5)\end{array}$ \\
$\begin{array}{l}\text { Score moyen Capacité } \\
\text { visuospatiale (sur 30) }\end{array}$ & $19(6,4)$ & $18,9(6,2)$ & $18,8(7,3)$ \\
$\begin{array}{l}\text { Fréquence des pratiques } \\
\text { médiatiques }\end{array}$ & $\begin{array}{c}41 \% \\
(10,1)\end{array}$ & $\begin{array}{c}39,5 \% \\
(10,9)\end{array}$ & $\begin{array}{c}39,7 \% \\
(10,9)\end{array}$ \\
\hline
\end{tabular}

Tableau 2 : Répartition des trois groupes expérimentaux

\section{Épreuves}

Les passations se sont déroulées au lycée pour des raisons pratiques d'accès aux techniciens novices, sur une période de deux mois. L'expérience consiste en quatre épreuves liées aux fonctions du matériel visuel rencontrées en maintenance aéronautique (information et mémorisation). Après pré-tests, les durées de ces épreuves ont été limitées pour éviter un effet «plafond » qui égalise les performances.

Les épreuves I et III visent à étudier la pertinence du contrôle utilisateur sur une tâche informative selon que la 3D montre (I) ou ne montre pas (III) un phénomène dynamique complexe. Dans l'épreuve I, les participants disposent de sept minutes pour manipuler l'animation 3D et reconstituer la procédure montrée en remettant dans l'ordre chronologique 12 vignettes provenant de cette animation. La même consigne est proposée pour les épreuves II et IV mais la reconstitution se fait de mémoire, sans support de l'animation, les mêmes 12 vignettes à ordonner ayant été placées dans de nouvelles configurations. La durée de ces épreuves est limitée respectivement à trois et deux minutes.

Entre ces deux rappels, dans l'épreuve III, les participants découvrent la modélisation 3D d'une vanne à air. L'épreuve commence lorsque sur un deuxième écran, 12 photographies sont affichées, donnant à voir des vannes sous différents angles et échelles. Le participant doit alors, en sept minutes, déterminer si chaque photo correspond ou non à la vanne modélisée.

\section{Matériels}

Pour les épreuves I, II et IV, le matériel visuel est une animation 3D décrivant comment démonter le moteur des essuie-glaces de l'A380 sous la forme d'un planséquence muet d'une minute trente. Les étapes de la procédure se manifestent par des arrêts de mouvements de caméra virtuelle, le clignotement des éléments concernés et l'apparition de mentions écrites minimales (en anglais). L'absence de montage oblige la caméra virtuelle à opérer des déplacements acrobatiques pour expliquer le mouvement complexe de désengagement de ce moteur.

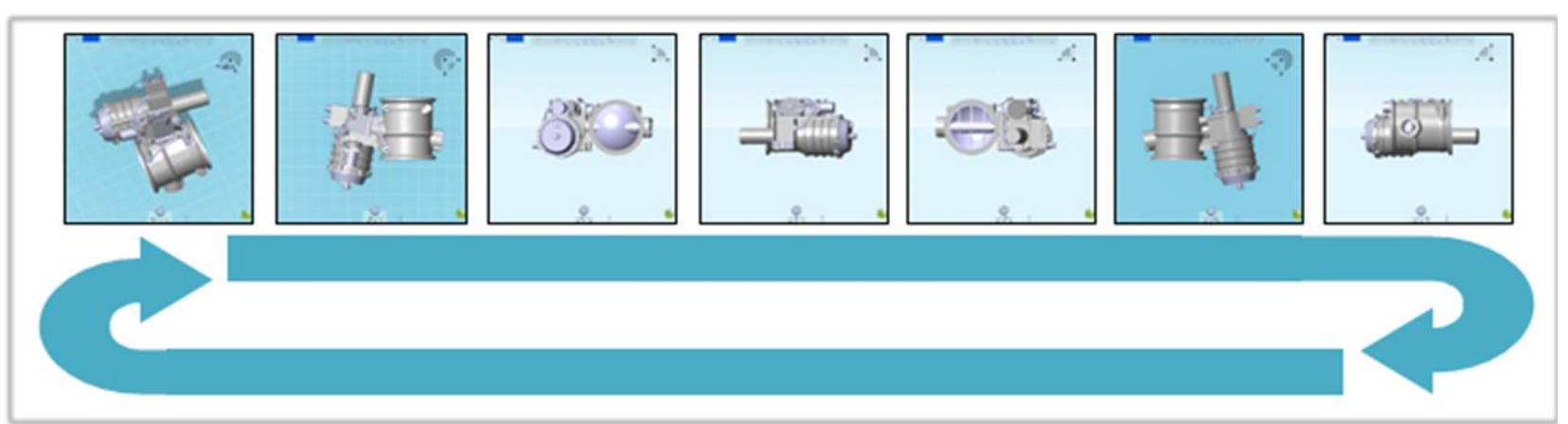

Figure 1 : Boucle animée de la vanne modélisée - épreuve III. 
Cette procédure animée peut être décomposée en 4 parties: accès à la pièce principale, déconnexion du moteur, mouvement de dégagement de celui-ci, sortie de la structure. Dans chaque partie, trois captures d'écran ont été réalisées pour obtenir douze vignettes en tout qui devront être réordonnées (Figure 2). Nous n'avons pas cherché à rendre l'épreuve plus difficile en proposant des vignettes résultant de la manipulation de la représentation linéaire (gros plans, angles de vues différents, etc.). Les mouvements du moteur sont matérialisés par des flèches rouges validées par deux experts. Il est précisé aux participants que ces flèches indiquent bien le déplacement de la pièce (et non celui de la caméra virtuelle).

Pour l'épreuve III, une visualisation 3D a été conçue par l'équipe d'EADS IW ; il s'agit d'une animation dans laquelle la pièce (vanne à air) opère des rotations successives afin d'être vue sous chaque angle de vue (Figure1). C'est sur cette séquence en boucle que s'effectuera le contrôle de la représentation. Deux moniteurs sont utilisés : l'écran de droite donne à voir l'animation puis la visualisation 3D ; l'écran de gauche délivre les consignes (Figure 3). Les participants répondent par écrit sur un formulaire, lequel porte également les quatre échelles NASA TLX à remplir après chaque épreuve.

\section{Mesures et recueils des données}

L'activité des épreuves de manipulation (I et III) est enregistrée. Le nombre et le type de commandes sont pris en compte, ainsi que le nombre de retour vers les photographies. Nous utilisons pour cela le logiciel TipExe [9] : les vues photographiques de l'épreuve III sont floutées et les participants doivent cliquer sur celles-ci pour les rendre nettes, le logiciel permettant ainsi l'obtention des durées exactes de prises d'information.

Pour les questions portant sur la restitution de la procédure (I, II et IV), nous proposons aux participants de séparer leurs réponses dont ils sont sûrs de celles plus incertaines. Chaque réponse fournit ainsi cinq données : l'exactitude de la réponse, le rang de la première erreur, le pourcentage d'éléments correctement placés, le rang à

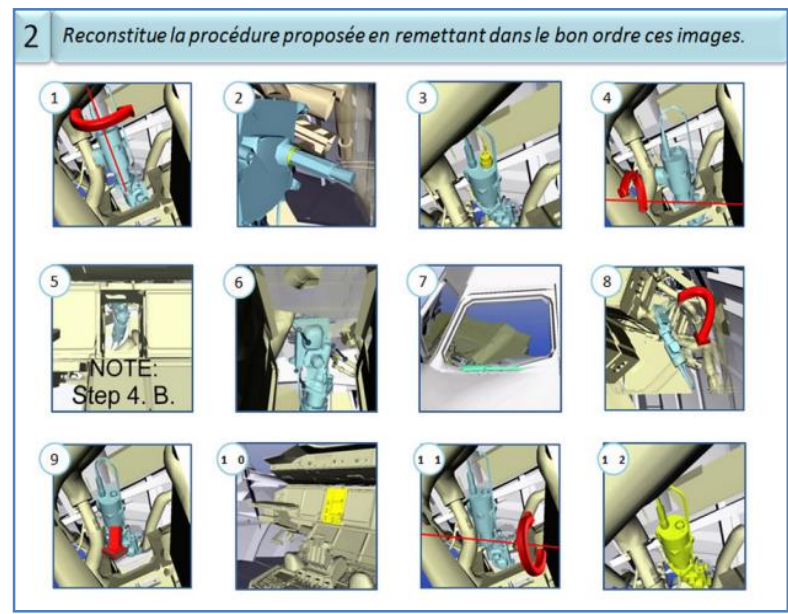

Figure 2 : Écran de consignes - épreuve II partir duquel commence la zone d'incertitude et enfin le pourcentage d'éléments sûrs d'après le participant.

Une appréciation de la certitude des réponses est également proposée pour l'épreuve III, sous la forme d'une échelle de Likert en 4 points apposée pour chaque réponse.

Enfin, après chaque tâche, l'utilisateur jauge la charge mentale fournie, avec l'échelle NASA TLX sur six critères : demande mentale, demande physique, exigence temporelle, sentiment de réussite, effort et frustration.

\section{Hypothèses}

Il est important de noter que dans l'épreuve I, l'utilisation des commandes de rotation, zoom et panoramique est absolument superflue : un participant avec la condition «contrôle étendu » se retrouve ainsi avec une batterie d'outils supplémentaires parfaitement inutiles pour mener à bien ce travail. Ces potentialités en excès peuvent gêner la bonne réalisation de l'épreuve I. Le curseur permettant les petits allers-retours nécessaires pour discriminer les mouvements du moteur, nous estimons que le groupe "médian» doit réaliser les meilleurs scores.

La réussite des épreuves de restitution (II et IV) va être conditionnée par la façon avec laquelle le participant a exploré le document 3D dans la première tache. Nous faisons donc l'hypothèse que le groupe médian dispose des meilleurs atouts pour effectuer ces deux reconstitutions.

L'épreuve III se décompose en plusieurs sous-opérations : (1) rendre net l'une des photographies proposées sur l'écran de gauche ; (2) en faire mentalement la description et en tirer une information discriminante pour l'identification ; (3) faire pivoter la représentation 3D pour permettre l'alignement le plus proche possible des deux vues ; (4) effectuer la comparaison ; (5) prendre la décision (oui/non) ; enfin (6) noter la réponse et formuler son degré d'engagement. Le degré de contrôle intervient donc précisément dans l'étape $n^{\circ} 3$ : les ressources cognitives engagées pour obtenir cet alignement ne peuvent pas être utilisées pour maintenir en mémoire de travail les deux représentations mentales dont on demande la comparaison.

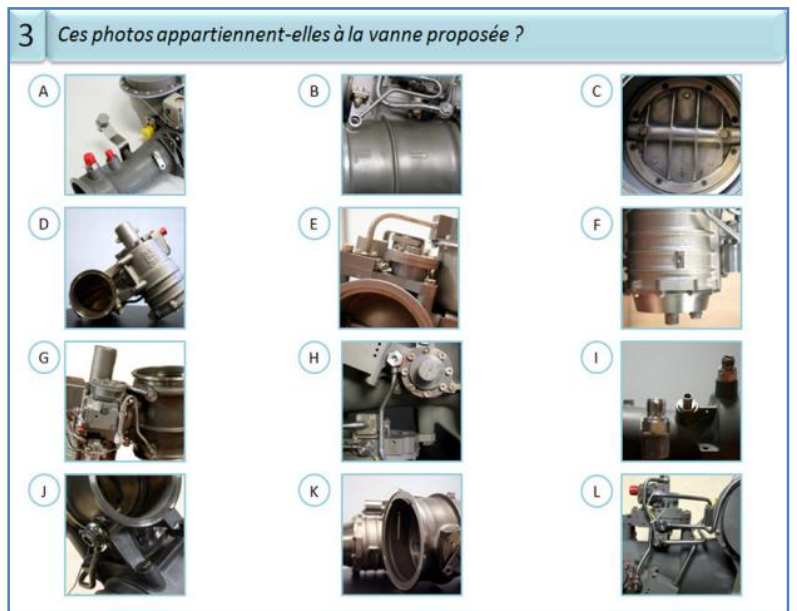

Figure 3 : Écran de consignes - épreuve III 
Plus cet alignement sera rapide à obtenir, plus l'opérateur sera aidé dans cette activité. C'est pourquoi nous pensons que les résultats de cet exercice seront meilleurs pour les participants qui disposent d'un contrôle étendu et d'une capacité VS élevée, mais que le contrôle limité favorisera les participants à capacité VS moins élevée.

\section{RÉSULTATS}

Prise d'information d'une animation 3D procédurale (épreuve I)

Le groupe au contrôle limité ne pouvant pas revenir en arrière, est désavantagé dans la gestion du temps et cela se retrouve dans la durée de l'épreuve (un tiers seulement termine avant les 7 minutes) (Tableau 3 ).

Pour l'exactitude des réponses (Tableau 4), le groupe « médian » obtient les performances les plus faibles : il donne moins de réponses correctes, se trompe plus tôt dans l'ordonnancement des vignettes et fait davantage d'erreurs multiples. Le groupe avec contrôle étendu réussit le mieux à reconstruire la procédure et est, dans le même temps, le plus confiant dans sa réponse. La différence de performances entre ces deux groupes, qui utilisent pourtant les mêmes commandes, est importante, notamment dans la restitution de la phase dynamique de la procédure $\left(\mathrm{X}^{2}(2 ; 43)=10,8 ; p=.005\right)$.
La comparaison des moyennes donne également des résultats significatifs d'un effet de la version sur le degré de certitude de la réponse $\left(\mathrm{F}_{(41)}=6,463 ; p=.004\right)$.

Par ailleurs une analyse des corrélations montre un lien entre capacité visuospatiale et rang moyen de la première erreur $(R(36)=, 386 ; \rho=.020)$.

\begin{tabular}{|l|c|c|c|}
\hline CONTRÔLE & Niv.1 & Niv. 2 & Niv.3 \\
\hline $\begin{array}{l}\text { Durée moyenne de l'épreuve (s) } \\
\text { (écart-type) }\end{array}$ & $\begin{array}{c}409 \\
(24)\end{array}$ & $\begin{array}{c}396 \\
(45)\end{array}$ & $\begin{array}{c}373 \\
(58)\end{array}$ \\
\hline $\begin{array}{l}\text { Nombre de participants } \\
\text { achevant l'épreuve avant 7 min }\end{array}$ & $5 / 15$ & $6 / 14$ & $8 / 14$ \\
\hline
\end{tabular}

Tableau 5 : Moyennes (écarts-types) des durées - épreuve I.

Pour réaliser l'épreuve, les $2^{\mathrm{e}}$ et $3^{\mathrm{e}}$ groupes actionnent les mêmes commandes comme le montre le très faible nombre de rotations et zooms réalisés par le groupe 3 (Tableau 5). Comment expliquer cet écart paradoxal de performances entre les deux groupes ? Le dénombrement des actions de commande réalisées suggère une piste : si l'activité est hétérogène pour les trois groupes, le groupe avec contrôle étendu a davantage exercé d'actions de commandes, ce groupe devançant même le groupe contrôle limité quant à l'utilisation de la touche [pause] (alors que ce premier groupe ne dispose que de celleci !). Si le second groupe a peu utilisé le déplacement

\begin{tabular}{|c|c|c|c|}
\hline CONTRÔLE & Niv.1 & Niv. 2 & Niv.3 \\
\hline $\begin{array}{l}\mathrm{Nb} \text { réponses correctes/attendues (pourcentages) } \\
\text { Procédure entière }\end{array}$ & $8 / 15(53,3 \%)$ & $5 / 14(35,7 \%)$ & $8 / 14(57,1 \%)$ \\
\hline $1^{\mathrm{e}}$ partie : accès pièce & $44 / 45(97,8 \%)$ & $42 / 42(100 \%)$ & $42 / 42(100 \%)$ \\
\hline $2^{\mathrm{e}}$ partie : déconnexions & $35 / 45(77,8 \%)$ & $31 / 42(73,8 \%)$ & $32 / 42(76,2 \%)$ \\
\hline $3^{\mathrm{e}}$ partie : mouvement moteur $*$ & $34 / 45(75,6 \%)$ & $21 / 42(50 \%)$ & $34 / 42(81 \%)$ \\
\hline $4^{\mathrm{e}}$ partie : sortie de la pièce & $31 / 45(68,9 \%)$ & $27 / 42(64,3 \%)$ & $36 / 42(85,7 \%)$ \\
\hline Rang moyen de la $1^{\text {ère }}$ erreur (écart-type) & $9,7(4)$ & $8,5(3,8)$ & $9,8(4)$ \\
\hline Exactitude de la réponse & $80 \%$ & $72 \%$ & $85,7 \%$ \\
\hline Rang moyen de perte de certitude (écart-type) & $8,7(2,7)$ & $8(3,2)$ & $10(3,3)$ \\
\hline Certitude de la réponse* & $68,9 \%$ & $63,1 \%$ & $89,9 \%$ \\
\hline
\end{tabular}

Tableau 3 : Exactitudes et certitudes des réponses - épreuve I (* résultats significatifs avec p<.05)

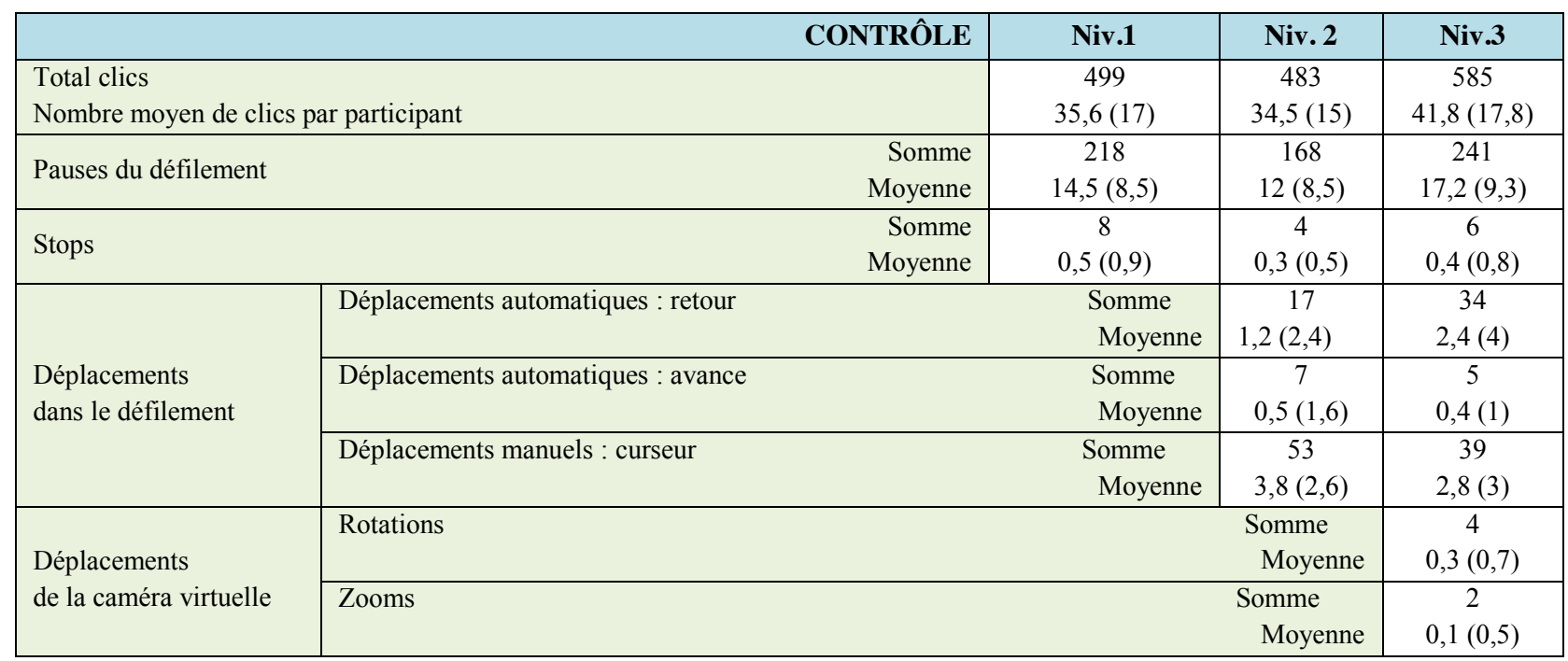

Tableau 4 : Nombres et moyennes (écarts-types) des types de commandes - épreuve I. 
automatique par rapport au troisième groupe (deux fois moins de [retour]s), il a davantage recouru au curseur pour se mouvoir dans la temporalité de la procédure Dans l'évaluation de la charge cognitive, les scores d'exigences mentales sont similaires (Tableau 6). Le sentiment de réussite se fixe par rapport au temps puisque c'est le seul élément objectif dont les participants disposent. L'analyse statistique confirme un lien significatif entre les versions proposées pour les sentiments de réussite $\left(\mathrm{F}_{(41)}=4,331 ; p=.020\right)$, et d'effort $\left(\mathrm{F}_{(41)}=4,636 ; p=.016\right)$

\begin{tabular}{|c|c|c|c|}
\hline CONTRÔLE & Niv.1 & Niv. 2 & Niv.3 \\
\hline Exigence mentale & $5,6(1,5)$ & $5,8(1,9)$ & $5,8(1,4)$ \\
\hline Exigence physique & $0,7(1,2)$ & $0,4(0,5)$ & $0,6(0,8)$ \\
\hline $\begin{array}{l}\text { Exigence } \\
\text { temporelle }\end{array}$ & $4,7(1,7)$ & $4,2(2,9)$ & $3,1(2,7)$ \\
\hline Réussite* & $5(1,8)$ & $4,1(2,4)$ & $6,4(1,7)$ \\
\hline Effort* & $3,5(1,8)$ & $4,8(1,6)$ & $2,9(1,6)$ \\
\hline Frustration & $3,6(1,9)$ & $2,5(2,2)$ & $2(1,7)$ \\
\hline
\end{tabular}

Tableau 3 : Moyennes (écarts-types) des évaluations de charge - épreuve I $(* \mathbf{p}<.05)$

\section{Mémorisation de la procédure (épreuves II et IV)}

Le groupe contrôle limité s'est montré globalement le plus rapide pour effectuer cette tâche de rappel la première fois, le groupe niveau 3 s'illustrant lui, lors de la seconde tentative (Tableau 7).

Si la séquence de 12 éléments était très difficilement mémorisable, le groupe avec contrôle limité est celui qui, en moyenne, se trompe le plus tardivement dans la réponse (au début du mouvement de la pièce), tandis que le groupe contrôle étendu se trompe le moins dans la reconstitution de ce mouvement complexe (45,2 \% contre $37,8 \%$ pour le groupe 1) (Tableau 8 ). Pour le groupe niveau 2, le taux d'exactitude se limite à 50\% avec un taux de rappel du mouvement réduit à $19 \%$. L'analyse statistique montre que le degré de contrôle a de nouveau influencé la restitution de la troisième partie de la procédure (le mouvement complexe) : $\mathrm{X}^{2}(2 ; 43)=$ $6,82 ; p=.033)$

\begin{tabular}{|c|c|c|c|c|c|c|}
\hline \multirow{2}{*}{ CONTRÔLE } & \multicolumn{3}{|c|}{ II } & \multicolumn{3}{|c|}{ IV } \\
\hline & Niv.1 & Niv. 2 & Niv.3 & Niv.1 & Niv. 2 & Niv.3 \\
\hline $\begin{array}{l}\text { Durée moyenne de l'épreuve (secondes) } \\
\mathrm{Nb} \text { de participants réussissant l'épreuve en }\end{array}$ & & $157(27)$ & $150(28)$ & $103(18)$ & $101(30)$ & $98(25)$ \\
\hline deçà de la durée prescrite & $13 / 15$ & $9 / 14$ & $11 / 14$ & $10 / 15$ & $10 / 14$ & $12 / 14$ \\
\hline
\end{tabular}

Tableau 7 : Moyennes (écart-types) des durées de réalisation - épreuves II et IV.

\begin{tabular}{|c|c|c|c|c|}
\hline & CONTRÔLE & Niv.1 & Niv. 2 & Niv.3 \\
\hline \multirow{8}{*}{ II } & $\begin{array}{l}\mathrm{Nb} \text { de réponses correctes/ attendues } \\
\text { Procédure entière }\end{array}$ & $1 / 15$ & $0 / 14$ & $0 / 14$ \\
\hline & $1^{\mathrm{e}}$ partie : accès pièce & $44 / 45(97,8 \%)$ & $39 / 42(92,9 \%)$ & $40 / 42(95,2 \%)$ \\
\hline & $2^{\mathrm{e}}$ partie : déconnexions & $27 / 45(60 \%)$ & $18 / 42(42,9 \%)$ & $22 / 42(52,4 \%)$ \\
\hline & $3^{\mathrm{e}}$ partie : mouvement moteur $*$ & $17 / 45(37,8 \%)$ & $8 / 42(19 \%)$ & $19 / 42(45,2 \%)$ \\
\hline & $4^{\mathrm{e}}$ partie : sortie & $24 / 45(53,3 \%)$ & $19 / 42(45,2 \%)$ & $24 / 42(57,1 \%)$ \\
\hline & Position moyenne de la $1^{\text {ère }}$ erreur (écart-type) & $6,3(2,8)$ & $4,9(1,7)$ & $5,4(1,9)$ \\
\hline & Exactitude de la réponse globale & $62,2 \%$ & $50 \%$ & $62,5 \%$ \\
\hline & $\begin{array}{l}\text { Rang moyen de perte de certitude } \\
\text { Certitude de la réponse }\end{array}$ & $\begin{array}{c}7,9(2,9) \\
64,4 \%\end{array}$ & $\begin{array}{l}6,4(3) \\
48,2 \%\end{array}$ & $\begin{array}{l}6,8(2) \\
60,7 \%\end{array}$ \\
\hline \multirow{8}{*}{ IV } & $\begin{array}{l}\mathrm{Nb} \text { de réponses correctes/attendues } \\
\text { Procédure entière }\end{array}$ & $1 / 15$ & $0 / 14$ & $0 / 14$ \\
\hline & $1^{\mathrm{e}}$ partie : accès pièce $*$ & $44 / 45(97,8 \%)$ & $34 / 42(81 \%)$ & $42 / 42(100 \%)$ \\
\hline & $2^{\mathrm{e}}$ partie : déconnexions $*$ & $32 / 45(71,1 \%)$ & $18 / 42(42,9 \%)$ & $21 / 42(50 \%)$ \\
\hline & $3^{\mathrm{e}}$ partie : mouvement moteur & $18 / 45(40 \%)$ & $10 / 42(23,8 \%)$ & $17 / 42(40,5 \%)$ \\
\hline & $4^{\mathrm{e}}$ partie : sortie & $19 / 45(42,2 \%)$ & $16 / 42(38,1 \%)$ & $24 / 42(57,1 \%)$ \\
\hline & Position moyenne de la $1^{\text {ère }}$ erreur (écart-type) & $6,6(2,8)$ & $5,3(2)$ & $5,5(1,7)$ \\
\hline & Exactitude de la réponse globale & $62,8 \%$ & $55,4 \%$ & $61,9 \%$ \\
\hline & $\begin{array}{l}\text { Rang moyen de perte de certitude } \\
\text { Certitude de la réponse }\end{array}$ & $\begin{array}{c}7,6(2,9) \\
61,7 \%\end{array}$ & $\begin{array}{l}6,6(3) \\
51,3 \%\end{array}$ & $\begin{array}{c}7,4(2,3) \\
67,9 \%\end{array}$ \\
\hline
\end{tabular}

Tableau 8 : Performances liées à l'exactitude - épreuves II et IV (* p<.05)

\begin{tabular}{|r|c|c|c|c|c|c|}
\hline & \multicolumn{3}{|c|}{ II } & \multicolumn{3}{|c|}{ IV } \\
\cline { 2 - 7 } CONTRÔLE & Niv.1 & Niv. 2 & Niv.3 & Niv.1 & Niv. 2 & Niv.3 \\
\hline Exigence mentale & $5,3(1,6)$ & $6,5(2)$ & $6,5(1,7)$ & $4,8(1,8)$ & $5,7(1,9)$ & $4,8(2)$ \\
Exigence physique & $0,7(1,6)$ & $0,3(0,5)$ & $0,5(0,6)$ & $0,6(1,2)$ & $0,4(0,6)$ & $1(1,2)$ \\
Exigence temporelle & $3,5(2,3)$ & $4,4(2,4)$ & $4,1(2,9)$ & $4,1(2,1)$ & $4,2(2,4)$ & $3,3(2,5)$ \\
Réussite & $5,2(1,9)$ & $3,1(2)$ & $4,8(1,4)$ & $4,7(1,6)$ & $4,1(1,7)$ & $5,2(1,8)$ \\
Effort & $3,7(2,3)$ & $5,6(2,1)$ & $3,7(2,1)$ & $3,9(2)$ & $4,6(1,9)$ & $3,5(1,8)$ \\
Frustration & $3,1(2,3)$ & $3(2)$ & $2,7(2)$ & $3,1(1,6)$ & $2,8(2,2)$ & $1,8(1,4)$ \\
\hline
\end{tabular}

Tableau 9 : Moyennes (écart-types) des évaluations de charge - épreuves II et IV. 
Pour le rappel différé, les participants avec contrôle limité ont davantage réussi : erreur plus tardive dans la réponse, taux global de vignettes bien placées. Il est suivi du groupe 3 qui affiche une plus grande confiance dans son résultat (près de $68 \%$ contre 61,7 pour le groupe 1 et $51,3 \%$ pour le groupe 2 ). L'analyse statistique montre que l'influence de la version ne se situe plus dans la phase dynamique mais dans les deux premières parties de celle-ci (respectivement $\mathrm{X}^{2}(2 ; 43)=14,1 ; p=.001$ et $\left.\mathrm{X}^{2}(2 ; 43)=7,6 ; p=.02\right)$. Une comparaison des moyennes obtenues montre que le nombre de clics effectués lors du contact avec le matériel dans l'épreuve précédente influence le rang de l'erreur et l'exactitude de la réponse pour le premier rappel (respectivement $\mathrm{F}_{(2,41)}=2,519$; $p=.047$ et $\left.\mathrm{F}_{(2,41)}=2,837 ; p=.030\right)$. Cette même donnée semble également influencer l'exactitude de la seconde restitution $\left(\mathrm{F}_{(2,41)}=2,567 ; p=.044\right)$. En revanche, aucune corrélation n'est établie entre la capacité visuospatiale et ces performances.

Les évaluations de la charge cognitive correspondent aux performances observées (Tableau 9). Pour le premier rappel, l'exigence mentale est jugée moindre pour le groupe 1 qui affiche aussi un plus grand sentiment de réussite mais également une frustration plus importante. L'effort, les demandes mentales et temporelles les plus importants se situent chez le groupe 2. Les choses ne changent pas avec le rappel différé pour le groupe 2 qui témoigne d'une charge plus forte (effort, demandes...). Pour le groupe avec contrôle limité, le degré de frustration demeure le plus élevé.

\section{Reconnaissance de pièces techniques (épreuve III)}

En moyenne, le groupe 2 est le moins rapide pour s'acquitter de cette épreuve (Tableau 10). On remarque cependant que, plus ils disposaient d'outils de commandes sur la représentation 3D, moins les participants ont investis de temps sur les photographies témoins ; 1'ANOVA réalisée indique un effet significatif de la version sur la durée de visionnement $(\mathrm{F}(41)=5,766$; $p=.006)$.

Sur l'ensemble des réponses, un écart dans le nombre de réponses correctes apparait entre le groupe contrôle étendu, et les autres versions, alors que le degré de certitude est proche pour les trois conditions matérielles. Cette influence n'est toutefois pas corroborée par des résultats statistiques.

L'analyse de l'activité produite (Tableau 11) montre un faible nombre de clics opérés par le groupe 1 (en moyenne 18 clics contre plus d'une trentaine pour les autres groupes), cette stratégie minimaliste n'étant pas sans réussite par ailleurs. Le groupe contrôle médian a privilégié les déplacements automatiques (alors qu'un déplacement manuel avec le curseur permettait d'observer davantage de positions intermédiaires). Le groupe contrôle étendu s'est massivement tourné vers la rotation ( 7 utilisateurs sur 14 , ne faisant quasiment que cette seule modalité de contrôle). Une analyse ANOVA montre une influence de la version proposée sur le nombre moyen de clics $\left(\mathrm{F}_{(41)}=4,845 ; p=.013\right)$, et sur le nombre d'utilisation du curseur $\left(\mathrm{F}_{(41)}=11,069 ; p<.001\right)$. Pour l'évaluation de la charge, l'exigence mentale augmente plutôt avec le niveau de contrôle quand le sentiment de réussite comme la frustration sont plus élevés avec la condition matérielle la plus restrictive (Tableau 12).

La capacité visuospatiale n'apparaît pas corrélée avec ces dernières évaluations. Des corrélations apparaissent cependant dans l'exactitude des réponses données pour 3 photographies sur les 12 de cette épreuve.

\begin{tabular}{|l|c|c|c|}
\hline \multicolumn{1}{|c|}{ CONTRÔLE } & Niv.1 & Niv. 2 & Niv.3 \\
\hline Durée de l'épreuve 3 $(\mathrm{s})(\max =420)$ & $374(67)$ & $392(46)$ & $391(40)$ \\
\hline $\begin{array}{l}\text { Nb de participants réussissant l'épreuve en deçà de la durée } \\
\text { prescrite }\end{array}$ & $9 / 15$ & $6 / 14$ & $9 / 14$ \\
\hline $\begin{array}{l}\text { Durée de visionnement des photographies (s) * } \\
\text { Temps de visionnement des photos/durée de l'activité }\end{array}$ & $\begin{array}{c}198,7(64) \\
52,5 \%(13,2)\end{array}$ & $\begin{array}{c}166,1(32) \\
42,3 \%(6,8)\end{array}$ & $\begin{array}{c}141(19,2) \\
36,2 \%(5,5)\end{array}$ \\
\hline Nb moyen de bonnes réponses & $8,9(2)$ & $8,9(1,8)$ & $9,8(1,5)$ \\
\hline Réussite dans l'identification & $74,4 \%(16,8)$ & $73,8 \%(15,3)$ & $81,5 \%(12,3)$ \\
\hline Certitude des réponses & $73,3 \%(11,2)$ & $69,9 \%(11,4)$ & $70,9 \%(9,1)$ \\
\hline
\end{tabular}

Tableau 10 : Moyennes (écarts-types) des performances - épreuve III $(* p<.05)$.

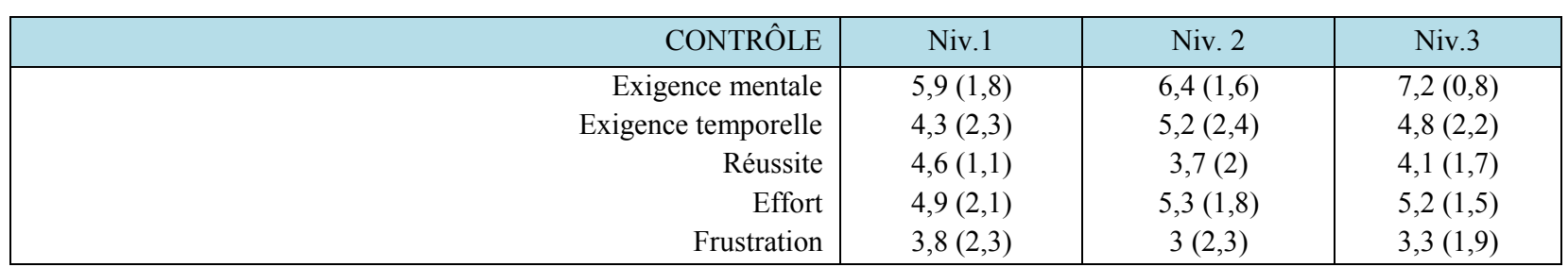

Tableau 12 : Moyennes (écarts-types) d'évaluations de charge - épreuve III. 


\begin{tabular}{|c|c|c|c|c|c|}
\hline & & CONTRÔLE & Niv.1 & Niv. 2 & Niv.3 \\
\hline \multicolumn{3}{|l|}{ Total clics } & 246 & 478 & 422 \\
\hline \multicolumn{3}{|c|}{ Nombre moyen de clics par participant* (écart-type) } & $17,6(18,9)$ & $34,1(14,8)$ & $30,1(8,6)$ \\
\hline \multirow{2}{*}{\multicolumn{2}{|c|}{ Pauses du défilement }} & Somme & 117 & 39 & 10 \\
\hline & & Moyenne & $8,4(9,3)$ & $2,8(2,4)$ & $0,7(0,7)$ \\
\hline \multirow{2}{*}{\multicolumn{2}{|c|}{ Stops }} & Somme & 24 & 42 & 10 \\
\hline & & Moyenne & $1,7(2,4)$ & $3(4)$ & $0,7(1,3)$ \\
\hline \multirow{6}{*}{$\begin{array}{l}\text { Déplacements } \\
\text { dans le défilement }\end{array}$} & \multirow{2}{*}{\multicolumn{2}{|c|}{ Déplacements automatiques : retour }} & Somme & 39 & 22 \\
\hline & & & Moyenne & $2,8(4,7)$ & $1,6(3,8)$ \\
\hline & \multirow{2}{*}{\multicolumn{2}{|c|}{ Déplacements automatiques : avance }} & Somme & 148 & 30 \\
\hline & & & Moyenne & $10,6(16,4)$ & $2,1(5)$ \\
\hline & \multirow[t]{2}{*}{ Déplacements manuels : curseur } & & Somme & 132 & 24 \\
\hline & & & Moyenne & $9,4(7,6)$ & $1,7(6,1)$ \\
\hline \multirow{6}{*}{$\begin{array}{l}\text { Déplacements } \\
\text { de la caméra virtuelle }\end{array}$} & \multirow[t]{2}{*}{ Rotations } & & & Somme & 284 \\
\hline & & & & Moyenne & $20,3(11,7)$ \\
\hline & \multirow[t]{2}{*}{ Zooms } & & & Somme & 12 \\
\hline & & & & Moyenne & $0,9(1,5)$ \\
\hline & \multirow[t]{2}{*}{ Déplacements } & & & Somme & 5 \\
\hline & & & & Moyenne & $0,4(0,9)$ \\
\hline
\end{tabular}

Tableau 11: Nombres et moyennes des types de commandes - épreuve III $(* p<.05)$

\section{CONCLUSION ET DISCUSSION}

Cherchant à mesurer l'influence du contrôle par les utilisateurs du matériel visuel $3 \mathrm{D}$, nous avons évalué celle-ci sur différentes tâches liées à la maintenance aéronautique. Cette évaluation portait sur les plans de l'utilité du document (exactitude des réponses) et de son acceptabilité (évaluation de la charge cognitive), lesquels sont liés à deux sources d'erreurs identifiées en maintenance, la non compréhension d'une procédure et son contournement.

Dans une première situation, une procédure animée comportant un nombre important d'étapes, montrait le mouvement particulièrement complexe d'une pièce technique à désengager et nécessitait donc une solide prise d'informations pour être correctement appréhendée ; dans un second cas, une boucle animée facilitait l'exploration visuelle d'un objet technique sous ses différents aspects. Pour chaque situation, des versions du matériel visuel selon trois degrés de contrôle ont été données à des groupes homogènes. Notre hypothèse initiale était que le niveau intermédiaire permettrait d'avoir suffisamment de contrôle sur la représentation pour appréhender et retenir les informations dynamiques sans dépenser trop de ressources cognitives dans la gestion de ce contrôle. Cette hypothèse générale n'est pas confirmée, bien au contraire.

Dans l'épreuve de compréhension de procédure (I), les meilleures performances ont été réalisées par les participants qui disposaient des commandes étendues : ils ont été plus rapides et ont donné davantage de bonnes réponses. Ils ont notamment fait moins d'erreurs dans la compréhension de la partie dynamique, ce qui va dans le sens du principe de segmentation proposé par Mayer \& Moreno [17].
Les participants avec contrôle de niveau intermédiaire obtiennent de moins bonnes performances, en-dessous même de celles réalisées avec contrôle limité. Le plus curieux est l'écart de résultats entre le groupe à contrôle médian et celui à contrôle étendu : ce dernier n'ayant pas utilisé tous les outils de commandes à sa disposition, les deux groupes étaient «à armes égales »; et pourtant l'un a de bien meilleures performances que l'autre. Or, ce qui distinguait ces deux groupes, c'était une possibilité de commandes, presque une liberté de choix. Et c'est ce luxe d'utilisation du document qui, semble-t-il, a conduit les participants de ce troisième groupe à avoir plus de contacts avec l'animation 3D (voir le nombre de clics), ce contact prolongé entraînant à la fois plus d'exactitude dans les réponses et des évaluations plus favorables de la charge cognitive, notamment l'effort. Ce n'est pas le fait d'avoir les bons outils qui a été déterminant, mais la possibilité donnée aux participants de pouvoir véritablement agir selon leur volonté. Pour caricaturer, dans cette épreuve l'acceptabilité pourrait avoir dominé l'utilisabilité.

Pour les deux tâches de rappels (II et IV), les résultats donnent un léger avantage au groupe avec le contrôle limité. Les efforts de compréhension réalisés dans la découverte de la procédure pour pallier le manque de contrôle, se sont avérés payants pour la création d'une représentation mentale plus exacte. Ce résultat pourrait donc être une illustration de la notion de «difficultés désirables » [27] lesquelles renforcent de manière contreintuitive les apprentissages à moyens et longs termes. Pour le rappel immédiat, le groupe avec contrôle étendu réussit cependant mieux la restitution de la partie dynamique de la procédure. De nouveau, ce qui paraissait être un juste calibrage de l'outil est dépassé par deux formes d'implications de l'opérateur: soit des 
efforts compensatoires pour réaliser la tâche, soit au contraire, un contact enrichi avec le document venant d'un sentiment de contrôle plus important. Dans le premier cas, l'opérateur agit pour combler un manque ; dans le second, l'opulence de moyens à sa disposition le motive (voir le ressenti de moindre frustration). Le niveau intermédiaire, dans ce cas, ne serait pas assez pauvre ou riche en contrôle pour créer l'une des deux situations d'implications. Si l'on adopte un point de vue lié à la théorie de Sweller sur la charge cognitive, le passage du niveau 1 au niveau 2, puis du niveau 2 au niveau 3 , pourrait ressembler au passage d'une situation de densité informationnelle importante, à une zone de faible interactivité des éléments (Interactivity elements effect) [29], pour repasser de nouveau dans une zone plus dense. Une situation comparable est décrite par Lowe [14] (underwhelming effect) lorsqu'un trop grand guidage d'une présentation dynamique entraîne une faible implication des apprenants. Cette tentative d'explication se heurte cependant à la très grande diversité d'activités enregistrées, entre des utilisateurs très économes en actions quand d'autres sont prolixes, quelle que soit la version du matériel proposé.

Pour la troisième épreuve, le gain octroyé par les commandes de rotation de la pièce était appréciable mais non déterminant puisque le déplacement du curseur permettait de retrouver les mêmes angles de vue. De nouveau, les meilleures performances sont obtenues par le groupe qui disposaient de la plus grande latitude de contrôle, mais au prix d'une plus grande exigence mentale. $\mathrm{Si}$, pour les performances les deux autres conditions ont donné des résultats comparables, le matériel intermédiaire a reçu des évaluations sur la charge ressentie les plus basses. De nouveau, il faut remarquer l'activité des participants hétérogène à l'intérieur de chaque groupe.

On pourra ensuite noter le faible nombre de corrélations impliquant la capacité visuospatiale sur les différentes mesures obtenues. L'influence déterminante de cette capacité n'a pas été démontrée, excepté dans la première épreuve (rang de la première erreur).

Les résultats de cette expérimentation, par rapport aux contenus et aux objectifs d'exploitation du document proposés en maintenance aéronautique, sont plutôt favorables aux animations avec contrôle étendu. Cette étude confirme aussi que le matériel le plus favorable aux performances n'est pas forcément celui qui sera le mieux apprécié par les opérateurs novices (risques de mauvaise interprétation vs. risques de défiance du document). De la même manière, un même document performant pour la prise d'information, le sera moins pour la mémorisation (voire l'apprentissage), ce qui limite la possibilité de documents visuels polyvalents. Un prolongement de l'étude avec des opérateurs experts du domaine serait pertinent : la population observée avait en effet des pratiques occasionnelles voire fréquentes de manipulation d'animations 3D; l'habitude du jeu vidéo (souvent corrélée avec certaines évaluations de charge) y était assez élevée, ce qui ne serait peut-être pas le cas avec une autre génération d'opérateurs. On pourrait également proposer une nouvelle session en laissant libre l'opérateur dans le choix des commandes afin d'analyser principalement les comportements d'utilisation : se limitent-ils à un seul type ou explorent-ils plusieurs outils ? Sont-ils économes ou au contraire, dépensent-ils beaucoup d'attention sur ce contrôle ? Est-ce que des stratégies seraient observables en fonction de paramètre de la tâche (temps à disposition par exemple) ? Enfin, l'activité de maintenance aéronautique étant réalisée par une équipe, il pourrait être pertinent de confronter un groupe à ces documents $3 \mathrm{D}$ afin de voir si les écarts observés avec la procédure prescrite sont régulées par la coopération.

\section{REMERCIEMENTS}

Les auteurs tiennent à remercier Laurent Juillac ainsi que les enseignants et étudiants du Lycée St Exupéry à Blagnac pour leur disponibilité.

\section{BIBLIOGRAPHIE}

1. Bétrancourt, M. The animation and interactivity principles, In Mayer R. (ed), the Cambridge handbook of multimedia learning, Cambridge University Press, Cambridge (2005), 287-296.

2. Bétrancourt, M., Tversky, B. Effect of computer Animation on users'performance: a review, Le travail Humain 63 (4) (2000), 311-330.

3. Bodemer, D., Ploetzner, R., Feuerlein, I., Spada, H. The Active integration of information during learning with dynamic and interactive visualizations, Learning and Instruction, 14 (3) (2004), 325-341.

4. Boucheix, J-M. On-line methods to study dynamic representations processing: eye tracking and comprehension. In proceedings of 12th Biennial Conference for Research on Learning and Instruction, Budapest, Hungary, August 28 September 1 (2007).

5. Boucheix, J-M. Control of multimedia Animations by children, Psychologie française, 53 (2008), 239-257.

6. Boucheix, J-M., Guignard, H. Which animation condition can improve text and comprehension in children?, Eur. J. Psychol. Educ., 20 (4) (2005), 369388 .

7. Durand, A., Laubin, J-M., Leleu-Merviel, S. Vers une classification des procédés d'interactivité par niveaux corrélés aux données, Hypertextes et hypermédias, 1(2-3-4), (1997), 367-382.

8. Evans, C., Gibbons, N. The interactivity effect in multimedia learning, Computers, Education, 49 (4) (2006), 1147-1160.

9. Ganier, F., \& Querrec, R. TIP-EXE; Editeur d'expériences et d'évaluations de documents procéduraux. Conférence internationale : "De la 
France au Québec : l'écriture dans tous ses états", Poitiers (2008), 40-42.

10. Hobbs A. An Overview of human factors in aviation maintenance, ATSB Safety Report, Aviation Research and Analysis Report AR 2008-055 (2008).

11. Hobbs A., Williamson A. Skills, rules and knowledge in aircraft maintenance: errors in context, Ergonomics 45 (4) (2002), 290-308.

12.Lattanzio, D., Patankar, K., Kanki, B. Procedural error in Maintenance: a review of research and methods, International Journal of Industrial Ergonomics 18 (1) (2008), 17-29.

13. Lombardo, E., Bertacchini, Y., Malbos, E. De l'interaction dans une relation pédagogique à l'interactivité en situation d'apprentissage, des théories aux implications pour l'enseignement (2006). Document consulté sur: isdm.univtln.fr/PDF/isdm\%2024/isdm24_lombardo.pdf

14. Lowe, R. Animation and learning: selective processing of information in dynamic graphics, Learning and Instruction, 13 (2) (2003), 157-176.

15. Lowe R. Interrogation of a dynamic visualization during the learning, Learning and Instruction 14 (2004), 257-274.

16. Mayer, R, Chandler, P. When learning is just a click away: does simple user interaction foster deeper understanding of multimedia messages?, Journal of Educational Psychology 93 (2001), 390-397

17. Mayer, R., Moreno, R. Nine ways to reduce cognitive load in multimedia learning, Educational Psychologist 38 (2003), 43-52.

18. McMillan, S. J., \& Hwang J. - S. Measures of Perceived Interactivity: An Exploration of Communication, User Control, and Time in Shaping Perceptions of Interactivity. Journal of Advertising (31) (2002), 41-54.

19. Muirhead, B., \& Juwah, C. Interactivity in computermediated college and university education: A recent review of the literature. Educational Technology \& Society 7 (1) (2004), 12-20.

20. Ossipow, B., Wipfli, R., Rebetez, C., Betrancourt, M. $\mathrm{Du}$ contrôle de l'Animation à la manipulation de contenu, JETCSIC 2006, Paris (2006).
21. Park, S., Lee, G., Kim, M. Do students benefit equally from interactive computer simulations regardless of prior knowledge levels?, Computers, Education, 52 (2009), 649-655.

22. Pognant, P., Scholl, C. Les CD-Roms culturels, Hermès, Paris (1996).

23. Rasch, T., Schnotz, W. Effects of animation's speed of presentation on perceptual processing and learning, Learning and Instructions 20 (2010), 136145 .

24. Rebetez, C., Bétrancourt, M., Sangin, M., Dillenbourg, P. Collaborer pour mieux apprendre d'une animation, IHM 2005, Toulouse (2005).

25. Rebetez, C., Bétrancourt, M. Faut-il vraiment prôner l'interactivité dans les environnements multimédias d'apprentissage ? Tecfa, Faculté de psychologie et des sciences de l'éducation hal-00161641, version 1 $11 \mathrm{Jul} 2007$.

26. Robin, D. Usages et bénéfices de la 3D en maintenance aéronautique. Thèse en Ergonomie, Université de Toulouse 2 (2012).

27. Robinson, D. Making tasks desirably difficult vs. Reducing extraneous cognitive load, $6^{\text {th }}$ International Cognitive Load Theory Conference, du 26 au 28 juin 2013, Toulouse (2013).

28. Schwan, S., Riempp, R. The cognitive benefits of interactive videos: learning to tie nautical knots, Learning and Instruction 14 (2004), 293-305.

29. Sweller, J. Instructional design in technical areas, Camberwell, Australia, ACER Press (1999).

30. Tassini, S., Bétrancourt, M. Effet du contrôle de l'animation sur son efficacité cognitive, 8ème journée JETCSIC 2003, Dijon (2003).

31. Wiesing, L. Réalité virtuelle : l'ajustement de l'image et de l'imagination «virtuelle Realität: die Angleichung des Bildes an die Imagination », in Artifizielle Präsenz. Studien zur Philosophie des Bildes, Francfort-sur-le-Main, Suhrkamp, 2005, 107124.

32. Zafiharimalala, H. Étude ergonomique pour la consultation sur écran de petite taille de la documentation de maintenance aéronautique, Thèse en Ergonomie, Université de Toulouse 2 (2011). 